\title{
Shy, withdrawn, or autistic?
}

\author{
Be worried when the parents are worried
}

Young children who are unusually shy or withdrawn may cause concern to parents or to the general practitioner, who finds them difficult to interview and examine. A reluctance to have too much to do with doctors is commonplace among young children (and indeed among adults), and the general practitioner who is confronted with a withdrawn clinging child must first consider whether the behaviour pattern is normal for the child's age and circumstances.

Intense attachment to a familiar adult begins to emerge at about 6 or 7 months and is accompanied by a fear of separation from the parent or other care giver and, commonly, a strong wariness of strangers. Clinging behaviour is increased if the child is ill, tired, or anxious. The intensity of these reactions to some extent reflects the child's genetically determined temperamental traits, modified by his or her experiences of relationships with familiar and unfamiliar people. The child's natural shyness of strangers may often be overcome by a gentle, patient approach. The toddler age group is the most difficult and the task gradually gets easier as the child reaches the age of 3 or 4 .

Shy or withdrawn behaviour is accepted as normal by most parents; playgroup and nursery teachers are perhaps even more tolerant of the wide range of normality in young children. Thus if parents are sufficiently worried to seek medical advice the problem deserves to be taken seriously.

It is important to establish exactly what is meant by "shy" or "withdrawn." Sometimes it means only that the child is slow to adjust to unfamiliar situations, perhaps taking months rather than days or weeks, to settle into a playgroup. This may simply reflect an introverted temperament, which may in retrospect have been recognisable since infancy. A parent may have had similar traits in childhood. If the child can play happily and relate normally to family members in familiar surroundings his or her shyness is probably a normal temperamental variant. This cannot be changed quickly or easily, but if the adults dealing with the child can alter their handling he or she may be able to adjust and adopt more effective styles of coping with the unfamiliar.

If, on the other hand, the behaviour causing concern is of recent onset or suggests a deterioration in social function a systematic history and a physical examination should first be undertaken to exclude physical illness. For example, it is easy to overlook secretory otitis media with fluctuant hearing loss.

There may be more serious reasons for withdrawn behaviour. In such cases the child's demeanour is likely to be sufficiently abnormal to worry the doctor even if the reason is not immediately apparent. He or she may be depressed because of worries about arrival of a sibling, bereavement, illness in a parent, or disturbed marital relationships (which the parents often wrongly imagine they have concealed from the child). There may even be emotional, physical, or sexual abuse; sometimes this results in the recognised clinical picture of the "frozen" child, who sits quietly but watchfully.

Withdrawn children may be miserable as a result of adverse circumstances or, rarely, their sadness may be complicated by self depreciation and inappropriate guilt, persisting even after adversity is corrected. In such cases clinical depression may reasonably be diagnosed. ${ }^{2}$ Such depression may be accompanied or masked by difficult and aggressive behaviour or physical symptoms, such as lack of interest, tiredness, and poor appetite. Parents occasionally conceal the information that would help the doctor to suspect this diagnosis, sometimes for perverse reasons, but more often to ensure that the doctor properly rules out physical disease first. Therefore a diagnosis of psychological illness may more profitably be suggested after the child has been physically examined than before.

If the child's withdrawn behaviour occurs within the family as well as with strangers, is not associated with low mood, and cannot be explained by disturbances in family circumstances, a communication disorder must be considered. Can the child speak normally for his age? Can he understand when spoken to by his parents? Does he show a desire to communicate and socialise? Does he play with parents or siblings? Does he indulge in meaningless, repetitive activities? Does he have problems in interacting with his peer group? If the parents express anxiety about any of these abilities the child may have a specific language impairment, Asperger's syndrome, autism, or elective mutism.

These conditions are not common, and few general practitioners will see more than one or two cases in their lifetime. Some doctors worry that they might miss such diagnoses, but this is unlikely provided that they listen to the parents' complaints. The child with a specific language impairment may have difficulties primarily with producing the individual sounds of speech, with grammar and vocabulary, or with the social rules of conversation. He will often also have impaired comprehension.

In autism not only language acquisition but also social behaviour are impaired. The child does not show normal attachment behaviour and does not relate normally to family members or peers, preferring his own company. He lacks the ability to play creatively or to pretend and has a tendency to 
sterile self occupation with monotonous routines. These features may have been present for as long as the parents can remember or may have had a sudden or gradual onset at any time in the first few years of life.

Asperger's syndrome is recognised by a stilted, eccentric use of speech and problems in understanding abstractions and metaphors, accompanied by pronounced social difficulties. These children may initiate social contact but they lack empathy and respect for the feelings of others, do not adapt to their interests, and are, understandably, short of friends. They seem socially odd and characteristically have rigid, sterile interests, such as electrical circuitry or bus routes, which cannot be shared with others. Clumsiness and an odd loping gait are common associated findings.

The relations between autism, Asperger's syndrome, and specific language impairment are still controversial, and the boundaries between them are not always clear. ${ }^{3}$ Selective (or elective) mutism, however, is a distinct entity. ${ }^{4}$ In contrast to these other disorders, it is commoner in girls. The child can talk and may do so freely at home but remains silent elsewhere. There may be an underlying speech and language impairment or mild learning difficulties, but the principal mechanism is usually an anxious avoidance of speech. Older children who refuse to speak are usually doing so out of anger.

All these conditions potentially retard development and educational progress, and any suspicion of them is an indication for a detailed assessment by a paediatrician or child psychiatrist. $^{5}$

D M B HALL Consultant in Community Child Health

P HILL Senior Lecturer in Child and Adolescent Psychiatry

St George's Hospital and Medical School.

London SW17 0QT

1 Hall DMB, Hill P, Elliman D. The child surveillance handbook. Oxford: Radcliffe Medical Press, 1990 .

Kazdin AE. Childhood depression. 7 Child Psychol Psychiatry 1990;31:121-60.

3 Green J. Is Asperger's a syndrome? Dev Med Child Neurol 1990;32:743-7.

4 Rutter M, Lord C. Language disorders associated with psychiatric disturbance. In: Yule W, Rutter M, eds. Language development and disorders. Oxford: MacKeith Press, 1987:206-33.

5 Byers-Brown B. Early identification of the language disordered child. In: Martin JM, Fletcher $\mathbf{P}$ Grunwell P, Hall DMB, eds. Proceedings of the first international symposium on specific speech and language disorders. London: Association for All Speech Impaired Children, 1987:135-44.

\section{Suicide in pregnancy and the puerperium}

\section{Much rarer now: thanks to contraception, legal abortion, and less punitive attitudes}

Retrospective studies of suicides suggest that at least $90 \%$ of victims were psychiatrically ill at the time of their death,,$^{12}$ and depression, schizophrenia, and alcohol dependence are all known to be associated with a high risk of self destruction. Such facts may lead psychiatrists and other doctors to assume that suicide risk is determined largely by mental state and psychiatric history. In reality, social factors are often of equal or greater importance, and nothing shows this more clearly than the effect of pregnancy and childbirth on suicide rates.

Using data from the Report on Confidential Enquiries Into Maternal Deaths from 1973 to 1984 Appleby has shown that in England and Wales the standardised mortality ratio for suicide - that is, the risk relative to that in other women of the same age - is only 0.17 in the first 12 months after childbirth and a mere 0.05 in pregnancy (p 137). Although he may have missed a few suicides in the early weeks of pregnancy and in the seventh to twelfth months after delivery, such losses are unlikely to account for these low mortality ratios. Indeed, Appleby's overall suicide rate in the puerperium is somewhat higher than that reported by previous authors. Barno found only $7 \cdot 7$ suicides per million births in the first postpartum year in Minnesota, ${ }^{4}$ and Kleiner and Greston only $5 \cdot 2$ suicides per million births in England and Wales in 1968-75. Appleby's 76 puerperium suicides represent 9.9 per million births.

Very low suicide rates in pregnancy, on the other hand, are a comparatively recent phenomenon. Kleiner and Greston compared 13 studies from several countries based on the years 1900-47 with 15 studies based on the years 1943-80. In the first period $12 \cdot 6 \%$ of all women of childbearing age who committed suicide were pregnant; in the second only $1.8 \%$ were pregnant. Weir was able to identify 66 pregnant women who had committed suicide, representing 59 per million live births, by searching the records of London coroners from 1943 to $1962 .{ }^{6}$ Over half died in the third to fifth months of pregnancy and a quarter in the third month. Sixty two per cent of women (and $82 \%$ of those dying in the third month) had illegitimate pregnancies, compared with $13 \%$ of all pregnant women. A generation later Appleby could find only 14 suicides in 12 years in the whole of England and Wales. This corresponds to 1.9 suicides per million births, just $3 \%$ of Weir's figure.

The reason for the low suicide rate in pregnancy and the puerperium is almost certainly, as Appleby suggests, that motherhood and the imperative social role this entails militate strongly against self destruction. ${ }^{3}$ The much higher standardised mortality ratio he found after stillbirth (1.05), and the fact that all four of the women who committed suicide after stillbirths were primiparous, is consistent with this explanation, as is the long established finding that the suicide rate of married women is lower in those with children than in those without. ${ }^{7}$ The virtual elimination of suicide in pregnancy over the past two generations is almost certainly due to a series of social changes that have greatly reduced the number of unwanted pregnancies and their adverse societal consequences - the increasing availability of effective contraception and of legal termination of unwanted pregnancies and the decreasing stigma attached to giving birth to children outside marriage. A high proportion of Weir's pregnant women, most of whom died before these changes occurred, had conceived outside marriage and took their lives in the third month of pregnancy, the stage at which two successive menstrual bleeds would have been missed. Attempted suicide used to peak in the third month for the same reason. ${ }^{8}$ Even now Appleby finds a significantly higher suicide rate in pregnant teenagers than in older women.

Despite the dominant role of social factors the influence of psychiatric illness is also evident, at least in the puerperium. The incidence of psychosis rises sharply in the first month after childbirth, with a risk relative to that in the year before the onset of pregnancy of over $20 .^{9}$ All of Barno's puerperal suicides occurred in the first three months after delivery and all 10 women were mentally ill. ${ }^{4}$ Eighteen of Appleby's 76 suicides occurred in the first four weeks after delivery. Although he had no information about the mental health of his subjects, the fact that so high a proportion killed 